\title{
Plastic Pre-Compression and Creep Damage Effects on the Fracture Toughness Behaviour of Type 316H Stainless Steel
}

\author{
Ali Mehmanparast ${ }^{1}$, Catrin M. Davies ${ }^{1}$, David W. Dean ${ }^{2}$, Kamran M. Nikbin ${ }^{1}$ \\ ${ }^{1}$ Mechanical Engineering Department, Imperial College London, South Kensington Campus, \\ London, SW7 2AZ. UK. \\ ${ }^{2}$ EDF Energy, Barnett Way, Barnwood, Gloucester, GL4 3RS, UK. \\ *Corresponding author: ali.mehmanparast@imperial.ac.uk
}

\begin{abstract}
The influence of inelastic damage in the form of plastic pre-strain and creep damage, on fracture toughness of Type $316 \mathrm{H}$ stainless steel has been examined. Creep damage has been introduced into the $8 \%$ pre-compressed material by interrupting creep crack growth tests. Comparisons have been made between the fracture toughness test results from the as-received, pre-compressed and creep damaged materials. Furthermore, the creep crack discontinuities effects on the crack tip strain fields have been examined by digital image correlation measurements. Inelastic damage was found to reduce the fracture toughness of the material, with creep damage having more severe effects than pre-strain.
\end{abstract}

Keywords: creep damage, fracture toughness, pre-straining, 316H stainless steel, micro cracks, crack discontinuity, DIC measurements

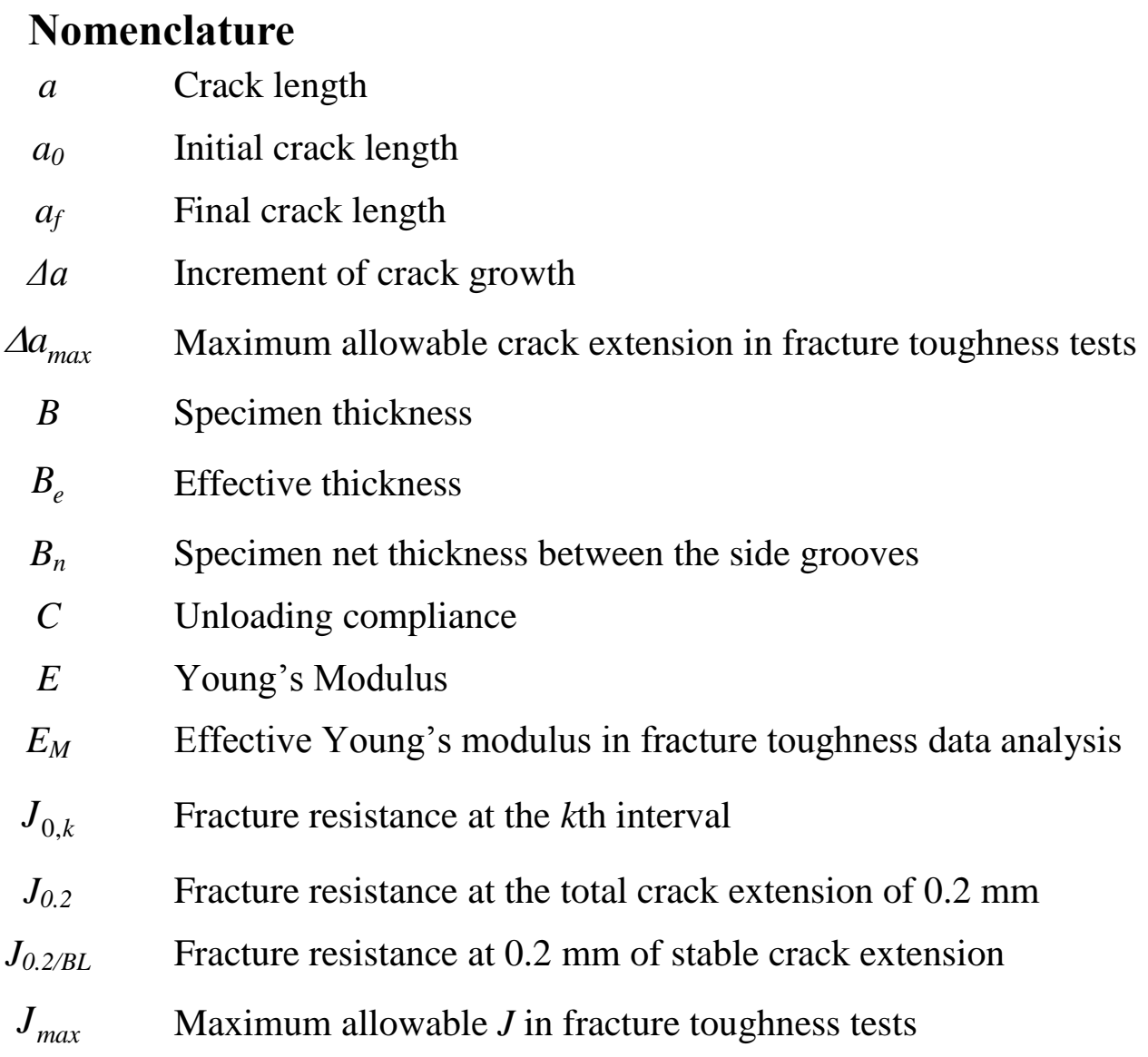




$\begin{array}{cl}J_{I C} & \text { Critical value of } J \text { for fracture under Mode I loading conditions } \\ K & \text { Stress intensity factor } \\ P & \text { Applied load } \\ P_{L C} & \text { Plastic collapse load } \\ U_{k} & \begin{array}{l}\text { Area under the force } v s . \text { displacement curve up to the line of constant displacement } \\ \text { at the } k \text { th interval }\end{array} \\ W & \text { Specimen width } \\ \eta & \text { Factor relating } J \text { to load and displacement measurements } \\ \sigma & \text { Applied stress } \\ \sigma_{0.2} & \text { 0.2 \% proof stress } \\ \sigma_{r e f} & \text { Reference stress } \\ \sigma_{U T S} & \text { Ultimate tensile stress } \\ \Omega & \text { Specimen size independency parameter in fracture toughness data analysis }\end{array}$

\section{Introduction}

Inelastic damage, in the form of both plastic strain and/or creep damage, is often present in components operating at elevated temperatures and may lead to changes in a material's mechanical response. Plastic strain can be introduced into a component in a number of ways during the fabrication process and creep strains/damage will accumulate with time at stress and temperature during component operation. Type $316 \mathrm{H}$ stainless steel (SS) is widely used in the UK's advanced gas cooled reactor's (AGR) high temperature components and therefore plays a key-role in securing the UK's short term energy supplies. Creep deformation and crack growth is the principal failure mechanism for these components, thus in order to increase plant life operating temperatures may be reduced to limit subsequent creep processes. However, in order to ensure structural integrity, the crack growth resistance of existing creep crack in components due to ductile damage process, i.e. the fracture toughness of the material, needs to be examined. Testing has therefore been performed to quantify the influence of inelastic damage on the fracture toughness behaviour of $316 \mathrm{H}$ stainless steel.

The influence of plastic pre-straining (introduced by cold rolling, pre-tension, precompression, etc) on fracture toughness of engineering materials have previously been examined. For example, it has been shown in [1] that the fracture toughness of an austenitic stainless steel continuously decreases as the level of plastic pre-straining increases from $0 \%$ to $15 \%$. A similar reduction in the fracture toughness of the pre-strained 316 material is also reported in [2]. Other studies of the influence of pre-straining on the fracture behaviour of a range of engineering materials can be found in e.g. [3-8]. The results obtained from these studies have shown that the fracture toughness generally decreases as the tensile or compressive percentage of plastic pre-staining increases in the material.

The influence of prior uniform creep damage on the subsequent tensile and fracture properties of 316 stainless steel has been investigated in [9] where creeping the material at $750{ }^{\circ} \mathrm{C}$ at $103 \mathrm{MPa}$ caused a moderate increase in the yield stress, severe reduction in tensile ductility and a rapid drop in the (Charpy) fracture energy. Experiments have also been performed in [10] on $316 \mathrm{H}$ at $550{ }^{\circ} \mathrm{C}$ and $300 \mathrm{MPa}$, where round bar creep tests were 
interrupted at different regions of the creep curve (i.e. primary, secondary or tertiary creep regions) and subsequently tensile tests were performed at a range of strain rates. The tensile ductility was found to be strongly dependent on both the quantity of creep strain introduced into the material and the strain rate of the tensile test. A similar study on $316 \mathrm{H}$ specimens which were pre-compressed to $8 \%$ plastic strain at room temperature and subsequently crept at $550{ }^{\circ} \mathrm{C}$ and $300 \mathrm{MPa}$ [11] has also confirmed that increasing the quantity of creep strain in the material increases its yield stress and decreases its tensile ductility. In addition an assessment of the effect of prior creep cavitation damage, arising from the stress relief heat treatment, on the measured brittle fracture toughness of a CrMoV steel weldment has shown that as the fraction of creep damage increases, a continuous reduction is observed in the fracture toughness of the material $[12,13]$. However, when the level of cavitation is relatively low, the fracture toughness of the creep damaged material has been found within the scatter band of the undamaged material. Literature therefore suggests that both plastic pre-straining and creep damage reduce the ductile/brittle fracture toughness of steels.

In this work, the influences of plastic pre-compression and creep damage on the subsequent fracture toughness behaviour of Type $316 \mathrm{H}$ Stainless Steel have been examined. Two methods have been considered to introduce creep damage into fracture mechanics samples. A large uniaxial geometry may be uniformly crept until a critical life/strain fraction is achieved and subsequently a fracture sample is extracted from this geometry. Alternatively, a creep crack growth (CCG) test on a fracture geometry may be performed and interrupted after a given amount of creep crack extension and hence creep strain/damage accumulation. The latter approach, denoted the local creep damage (LCD) method, provides a creep process zone local to the crack tip and has been employed in this work.

\section{Material and Specimen Test Details}

For the present study, Type $316 \mathrm{H}$ stainless steel was provided by EDF Energy and taken from an ex-service steam header. All tests been performed on the standard compact tension, $\mathrm{C}(\mathrm{T})$, geometry. The tensile and creep properties of this header material have been previously characterised as detailed in [14].

Type $316 \mathrm{H}$ SS has a relatively low yield stress at typical CCG testing temperatures of around $550{ }^{\circ} \mathrm{C}\left(\sigma_{y} \approx 170 \mathrm{MPa}[14]\right)$. Hence the development of some plastic strain during the specimen loading and unloading at this temperature is inevitable. Interpreting the results from interrupted CCG test data obtained from as-received (AR) material will therefore be complicated due to the difficulties to distinguish between the influence of creep and plasticity on the material's fracture behaviour. However, it has been previously shown that the extent of plasticity on loading is significantly reduced when the material is pre-compressed to $8 \%$ plastic strain at room temperature [14]. Therefore, to examine the influence of the extent of creep damage, whilst minimising the variation in crack tip plasticity in the samples, the material for LCD tests has been pre-conditioned through uniform pre-compression (PC) to $8 \%$ plastic pre-strain at room temperature, thus hardening the material.

In order to understand the effects of the uniform pre-compression alone on the fracture behaviour of the material, fracture toughness tests have been performed and compared on samples manufactured from AR and PC materials in addition to LCD samples. Note that the interrupted CCG tests to produce the LCD samples were conducted at $550{ }^{\circ} \mathrm{C}$, however to avoid any ambiguity in the data due to any variations in temperature during testing and creep strains that may accumulate in fracture toughness tests at elevated temperatures, all fracture toughness tests were performed at room temperature. 
Five $\mathrm{C}(\mathrm{T})$ specimens with the loading direction parallel to the pre-compression axis were extracted from the pre-strained blocks. Three fracture toughness tests have been performed on LCD samples, two on AR and two on PC material. The AR and PC specimens were prefatigue cracked prior to fracture toughness testing to introduce sufficiently sharp crack tip into the samples. After pre-cracking, the AR and PC samples were side grooved to $20 \%$ of the total thickness.

\section{Fracture Toughness Testing and Analysis Methodology}

$316 \mathrm{H}$ is a ductile material therefore its fracture toughness is determined via $J_{I C}$ testing. In ductile materials, three stages are often observed prior to the final fracture [15]:

i. Formation of a free surface at a particle/inclusion and nucleation of voids

ii. Void growth due to plastic strain and hydrostatic stress

iii. Void coalescence and formation of the micro-cracks

In service exposed metals, pre-existing voids can be usually observed in the material. Therefore the failure of a component is mainly controlled by void growth and coalescence stages. There are various models to calculate the void growth and coalescence in ductile materials. Rice and Tracey model [16] is the one which is commonly used to assess the void growth rates in ductile materials.

Various standard test methods are available for measuring fracture toughness of materials by performing tests on fracture mechanics specimens. The ones which are most widely used are the ASTM standard E1820 [17] and ESIS P2-92 [18]. The fracture toughness tests in this work have been conducted on $\mathrm{C}(\mathrm{T})$ specimens following the ESIS P2-92 procedure [18]. A summary of the fracture toughness testing and analysis method for ductile materials with stable crack extension is described below.

\subsection{Generation of the R-Curve}

In ductile materials, if the force $v s$. displacement behaviour of a cracked body is non-linear and stable crack growth is observed, the variation of $J$ can be plotted against the crack extension $\Delta a$. The $J v s$. $\Delta a$ variation is generally referred to as resistance curve (R-curve). The single specimen fracture toughness testing approach has been employed in this work where the specimen is frequently loaded and unloaded (to approximately $20 \%$ of the load peak) during the test and the instantaneous normalised crack length, $a / W$, is approximated using the unloading compliance data by

$$
a / W=\left(\begin{array}{l}
1.000196-4.06319 \mu+11.242 \mu^{2} \\
-106.043 \mu^{3}+464.335 \mu^{4}-650.677 \mu^{5}
\end{array}\right)
$$

where

$$
\mu=\frac{1}{\sqrt{B_{e} E_{M} C}+1}
$$

In Eqn (2) $C$ is the elastic unloading compliance, $E_{M}$ is the effective Young's modulus which can be calculated using the equation given in [18] and $B_{e}$ is the effective thickness which can be calculated by

$$
B_{e}=B-\left(B-B_{n}\right)^{2} / B
$$

where $B$ is the total thickness and $B_{n}$ is the net thickness between the side grooves. To account for the changes in the specimen geometry, which can occur due to loading, the measured compliances were corrected considering the rotation formulae given in [18].

The fracture resistance at the $k^{\text {th }}$ interval, $J_{0, k}$, can be calculated by 


$$
J_{0, k}=\frac{\eta U_{k}}{B_{n}\left(W-a_{0}\right)}
$$

where $a_{0}$ is the initial crack length, $W$ is the specimen's width, $U_{k}$ is the area under the force $v s$. displacement curve up to the line of constant displacement at the $k$ th interval and $\eta$ is a geometry dependent parameter [19]. After the final unloading, the load is reduced to zero and the sample is heat tinted to mark the final crack length. Subsequently the specimen is broken open to measure the final crack length using the digital imaging technique [20].

\subsection{Crack Growth Limits}

An R-curve is generated by plotting $J$ values against the corresponding crack extensions, $\Delta a$. In order to identify valid data points for fracture toughness analysis, the crack growth limits must be applied by specifying the slope of the blunting line (also known as construction line) and constructing the crack growth exclusion lines parallel to the blunting line at the offsets corresponding to the maximum allowed crack extension, $\Delta a_{\max }$, and $0.10 \mathrm{~mm}$, where $\Delta a_{\max }$ can be calculated by

$$
\Delta a_{\text {max }}=0.10\left(W-a_{0}\right)
$$

and the details of the blunting line slope calculations are given in ESIS P2-92 standard [18]. The data points falling in between exclusion lines are considered valid and the rest assumed invalid. The equation of the line of best fit must be determined considering the valid data points which lie between $0.1 \mathrm{~mm}$ and $\Delta a_{\max }$ exclusion lines on the $J v s . \Delta a$ graph.

\subsection{J Validity Limits}

To determine material's fracture behaviour independent of the specimen size, an exclusion line must be constructed on the $J v s$. $\Delta a$ graph at $J_{\max }$ value which can be calculated by

$$
J_{\max }=\operatorname{Min}\left\{\left(W-a_{0}\right)\left(\sigma_{0.2}+\sigma_{U T S}\right) / 40 ; B\left(\sigma_{0.2}+\sigma_{U T S}\right) / 40\right\}
$$

where $\sigma_{0.2}$ is $0.2 \%$ proof stress which is often taken as the yield stress of the material and $\sigma_{U T S}$ is the ultimate tensile strength (UTS) of the material. Subsequently, $\Omega$ parameter must be determined from

$$
\Omega=\frac{W-a_{0}}{J_{g}} \frac{d J}{d(\Delta a)} \mid J_{g}
$$

where $\frac{d J}{d(\Delta a)}$ is the differentiation of the equation of the line of best fit made to the valid data points on the R-curve. In Eqn (7) $\frac{d J}{d(\Delta a)}$ should be evaluated at $J_{g}$. Note that $J_{g}$, which is defined as the upper limit to $J$-controlled crack growth behaviour for the test specimen size, can be provisionally taken as the intersection of the best fit curve with either $J_{\max }$ or $\Delta a_{\max }$ exclusion lines. If $\Omega \geq 10$, the fracture resistance curve enclosed by $J_{\max }$ and $\Delta a_{\max }$ exclusion lines may be considered as material property independent of the specimen size.

\subsection{J Fracture Parameters}

The $J_{0.2 / B L}$ fracture parameter, which provides an engineering definition of crack initiation and measures the material resistance at $0.2 \mathrm{~mm}$ of stable crack extension, can be calculated by constructing a line parallel to the blunting line at the offset of $0.2 \mathrm{~mm}$. The intersection between this line and the best fit curve to the valid fracture resistance data points is regarded as $J_{0.2 / B L}$. Note that at least one data point should exist between this offset line and the $0.1 \mathrm{~mm}$ exclusion line. $J_{0.2 / B L}$ is only valid if, 


$$
\begin{gathered}
J_{0.2 / B L}<J_{\max } \\
\text { and } \\
(d J / d a)_{B L}>2(d J / d a)_{0.2 / B L}
\end{gathered}
$$

where $(d J / d a)_{B L}$ is the slope of the blunting line and $(d J / d a)_{0.2 / B L}$ is the slope of the best fit curve at $J_{0.2 / B L}$.

In addition, the $J_{0.2}$ fracture parameter, which provides a measure of material resistance at $0.2 \mathrm{~mm}$ total crack growth, can be evaluated by constructing a vertical line at the total crack extension of $0.2 \mathrm{~mm}$. The intersection between this line and the best fit curve to the valid fracture resistance data points on the $J v s$. $\Delta a$ curve is defined as $J_{0.2}$. Note that at least one data point should exist between $0.2 \mathrm{~mm}$ and $0.4 \mathrm{~mm}$ crack extension and $J_{0.2}$ is only valid if it's smaller than $J_{\max }$.

\section{Local Creep Damage Tests}

In order to introduce creep damage local to the crack tip, CCG tests were performed on three $\mathrm{C}(\mathrm{T})$ specimens made of PC material with the initial normalised crack length of $a_{0} / W=0.35$ which were subsequently interrupted at the approximate normalised crack length of $a / W \approx 0.5$. The LCD tests were performed at a load corresponding to an initial stress intensity factor of $K\left(a_{0}\right)=25.5 \mathrm{MPa} \sqrt{\mathrm{m}}$, at which crack tip plasticity effects on the CCG behaviour are limited for $a / W<0.5$.

Two CCG tests were initially performed on $\mathrm{C}(\mathrm{T})$ specimens with nominally identical geometries and material properties to the three LCD specimens, which are here denoted 8PC$\mathrm{A} 2$ and $8 \mathrm{PC}-\mathrm{A} 3$. The results from these two tests, which are referred to as CCG calibration tests, were used to characterise the direct current potential drop (DCPD) response to CCG length for this material and hence used to estimate the attainment of a normalised crack length of around $a / W \approx 0.5$ in the LCD samples. Note that the same load was used in the calibration tests and the LCD tests as plasticity is known to affect the response of the DCPD measurement [21].

The three LCD tests were interrupted, at the earliest convenient time, when the PD rates, reached values corresponding to $a / W=0.5$ in the $C C G$ calibration tests. The PD rate data $d P D / d t$, are presented in Figure 1. In this figure the CCG calibration tests and LCD data are shown in grey and black symbols, respectively. Also seen in this figure are the solid and dashed grey lines for the $\mathrm{PD}$ rate data corresponding to the normalised crack length of $a / W=0.5$ in the $8 \mathrm{PC}-\mathrm{A} 2$ and $8 \mathrm{PC}-\mathrm{A} 3 \mathrm{CCG}$ calibration tests, respectively (which were evaluated post testing by breaking open 8PC-A2 and 8PC-A3 specimens and calibrating the PD data).

As seen in Figure 1, different PD rates have been attained in 8PC-A2 and 8PC-A3 tests at $a / W$ of 0.5 which may be attributed to a number of factors including precise probe location [21]. Note that due to the large acceleration in CCG rates of PC material at larger crack extensions, it was difficult to interrupt the LCD tests at precisely the same crack lengths. The most conservative measure of PD rate obtained from specimen 8PC-A2 was used to interrupt the LCD tests. As seen in Figure 1, the LCD tests were stopped at the PD rate of around $5 \times 10^{-4}(\mathrm{mV} / \mathrm{h})$ which corresponds to the $a / W=0.5$ in the CCG calibration test on 8 PC-A2 sample. The actual final creep crack length was measured subsequent to fracture toughness test completion on the LCD samples by breaking open the samples. The measured creep crack lengths in LCD specimens, $a_{c}$, are summarised in Table 1 and taken as the initial crack length, $a_{i}$, in subsequent fracture toughness tests. 


\subsection{Residual Stresses}

During a CCG test, the specimen is loaded in tension at $550^{\circ} \mathrm{C}$ and unloaded at the end of the test after some amount of crack extension. The process of loading (i.e. pre-tension), crack propagation and unloading may lead to a compressive residual stress (RS) field being generated at the crack tip, which may subsequently affect the fracture toughness results on LCD specimens. Neutron diffraction (ND) measurements have been performed on 8PC-A2 and LCD specimens to quantify the extent of residual stresses ahead of the final crack tip subsequent to CCG test completion and prior to fracture toughness testing. The measurement details and results have been presented in [22, 23]. A compressive residual stress of around $-100 \mathrm{MPa}$ was observed at the final crack tip position in the direction normal to crack plane in all LCD samples. Note however that during fracture toughness testing, these stresses are expected to be soon eliminated and thus have little or no influence on the result.

\section{Fracture Toughness Tests}

Standard fracture toughness tests have been performed on two AR (JIC-AR1, JIC-AR2), two PC (JIC-PC1, JIC-PC2) and two LCD (LCD1, LCD2) specimens at room temperature, following the ESIS P2-92 standard as detailed in Section 3. All of these samples were sidegrooved as detailed in Table 2 where specimen dimensions, the through thickness averaged initial, $a_{i}$, and final, $a_{f}$, crack lengths are given. The $a_{f}$ measurements were made post testing by heat tinting (to mark the final crack tip) and measuring the crack area, as detailed in [20]. In sample LCD3, the side-grooves were machined off after CCG testing to easily facilitate the crack tip strain fields to be observed during fracture toughness testing using the digital image correlation (DIC) as will be later described.

As seen in Table 2, the average initial and final crack lengths, normalised by the samples' width, are in the range of $0.49-0.55$ and $0.55-0.64$, respectively, for the specimens tested. This means that all the fracture toughness samples were tested within the valid range of $0.45 \leq a / W \leq 0.65$ as recommended in the ESIS P2-92 standard [18]. Also seen in Table 2 is that the AR and PC specimens were side grooved by $20 \%$ of the specimen thickness according to the ESIS P2-92 standard. However due to the limit of the maximum load carrying capacity of the creep machines and to perform interrupted CCG tests on LCD specimens in a reasonable time, the LCD specimens had larger side grooves ( $30 \%$ in total). This difference between the net thickness of LCD specimens compared to AR and PC samples is expected to have insignificant effects on the fracture toughness results since, as later shown, specimen size independency criterion was achieved.

\subsection{Fracture Resistance Curves}

The load vs. load line displacement (LLD) data recorded during the fracture toughness tests are shown in Figure 2, which includes the loading/unloading data used to calculate the elastic compliance and subsequently instantaneous crack length. The linear response in the elastic part of the load vs. LLD trends for the AR (i.e. JIC-AR1 and JIC-AR2), PC (i.e. JIC-PC1 and JIC-PC2) and LCD (i.e. LCD1 and LCD2) specimens are similar, as expected. Figure 2 shows that for a given load, a greater LLD is achieved in the AR material compared to the PC and LCD samples. This is due to the lower yield stress of the AR material. Also seen in this figure is that, due to the hardening effects, a larger maximum load, $P_{\max }$, is observed in PC specimens during the fracture toughness tests compared to the AR specimens. However, due to the creep damage effects in LCD samples, the $P_{\max }$ measured in the LCD specimens falls below those of seen in PC samples. Note that the LCD specimens were previously precompressed to $8 \%$ plastic strain at room temperature, hence the dislocation structures have been changed, compared to the AR material, and some small initial voids may have been 
produced. During creep tests at higher temperature, these small initial voids promote creep damage, in addition the yield strength decreases compared to the PC material (see Figure 2) and the microstructure changes due to the tempering. These effects may lead to the lowest fracture resistance being observed in the LCD specimens.

The fracture resistance curves, R-curves, for the tests performed on the AR, PC and LCD specimens are presented in Figure 3. Note that the crack extensions presented in this figure were approximated using Eqn (1) employing the unloading compliances observed in Figure 2. In Figure 3, the AR specimens are shown in solid symbols whereas the PC and LCD samples are shown in hollow symbols. For a given crack extension, $\Delta a$, the energy absorbed during fracture in the AR material is significantly greater than that for the PC. This is thought to be due to the higher yield stress and lower tensile ductility in the PC material compared to AR, as shown and discussed in [14]. In other words, although the pre-compression process increases the yield stress of the material effectively by increasing the dislocation density, the formation of small voids may facilitate the fracture of the material and thus a lower fracture resistance is observed in the PC material.

The R-curve trends in the LCD samples for $\Delta a>3.5 \mathrm{~mm}$ are similar to that seen in the PC samples (Figure 3). For $\Delta a<3.5 \mathrm{~mm}$ in the LCD data, the $J$ value measured is low and relatively independent of crack length, this region is here denoted the 'tail'. This region is considered to comprise of extensive, discontinues creep damage, which consists of intergranular micro cracks and thin, weak unbroken ligaments. The fracture toughness results from the AR, PC and LCD samples are next presented and compared with each other.

\subsection{Fracture Toughness Determination}

The methodology for quantifying the fracture toughness value is exemplified here for sample JIC-AR1. The maximum valid crack extension, $\Delta a_{\max }$, and the slope of the blunting line have been calculated for each test and the results are tabulated in Table 3. Note that the specimen dimensions required for analysing the fracture toughness tests data from the AR, PC and LCD specimens have been taken from Table 2. Furthermore, the tensile data employed in the data analyses were taken from [14].

As seen in Figure 4(a), the blunting line has been constructed and the exclusion lines, which are parallel to the blunting line with an offset of $0.1 \mathrm{~mm}$, and $\Delta a_{\max }$ have been plotted on the $J v s . \Delta a$ graph. The data points fall in between these two exclusion lines are considered valid and shown in black symbols. However, the invalid data behind the $0.1 \mathrm{~mm}$ or in front of the $\Delta a_{\max }$ exclusion lines are shaded in grey. After applying the fracture resistance validity criteria, the line of best fit has been made to the valid data points as seen Figure 4(a). When the valid data points are verified, the maximum allowable $J$ value, $J_{\max }$, and subsequently the $\Omega$ parameter have been calculated using Eqn (6) and Eqn (7), respectively, and the results are summarised in Table 3. As seen in Table 3, the value of $\Omega$ parameter for the fracture toughness test on JIC-AR1 specimen is larger than 10, thus the valid data points which fall below the $J_{\max }$ exclusion line may be considered a material property, independent of the specimen size. Furthermore, the construction lines for all other validity criteria detailed in Section 3 are shown in Figure 4(b), for example, and the relevant validity criteria and fracture toughness parameter values for all test samples are given in Table 3.

\subsection{Pre-compression Effects on Fracture Toughness}

As seen in Table 3, the slopes of the blunting lines have been found very similar in the AR and PC materials. Though slightly larger values of $J_{\max }$ are generally observed in the specimens made of AR material compared to the PC samples. The values of $\Omega$ calculated for the tests on $\mathrm{AR}$ and $\mathrm{PC}$ specimens have been found larger than 10 and therefore the valid data 
points which are below $J_{\max }$ in each of these tests can be considered independent of the specimen size.

Further seen in Table 3 is that the $2 d J / d \Delta a_{(0.2 / B L)}$ values are smaller than the calculated slopes of the blunting lines and also $J_{0.2 / B L}$ values fall below $J_{\max }$ in each of these tests examined on the AR and PC materials. This means that the $J_{0.2 / B L}$ values found in these tests are valid. Finally observed in Table 3 is that all the $J_{0.2}$ values found for the tests on AR and PC materials can be considered valid since they are below the corresponding $J_{\max }$ for each test. Comparison of the values of fracture toughness parameters obtained from the tests performed on the AR and PC materials show that $J_{0.2 / B L}$ and $J_{0.2}$ values are larger in the AR material compared to the PC. This means that greater amount of fracture energy is needed to initiate the crack in the AR material due to the larger failure strain compared to the PC material. The observed reduction in the fracture toughness of the compressively pre-strained material to $8 \%$ plastic strain, compared to the as-received material, is consistent with the data observed in the literature e.g. [1-3].

\subsection{Creep Damage Effects on Fracture Toughness}

Microscopic images of the CCG behaviour in $316 \mathrm{H}$ stainless steel, captured at the midthickness of a PC specimen, have been shown in Figure 5 and discussed in [14]. Discontinuous cracks were observed at the plane considered that did not link up with the initial EDM notch. Hence, contrary to the standard fatigue pre-crack often introduced into fracture toughness tests, which generates two disconnected surfaces, the creep pre-crack present in the LCD samples was discontinuous and contained unbroken ligaments along the crack length. As noted in Section 3.1, during the fracture toughness test the crack length is measured using the compliance technique. Due to the nature of the discontinuous creep cracks in the LCD samples, the exact position of the crack tip that is fully connected to the initial EDM notch is unknown. To examine the extent of discontinuous cracking and its influence on the fracture toughness result a fracture toughness test was also performed on a plain sided sample, denoted LCD3, that enabled the surface strain fields to be mapped during sample loading employing the DIC technique. During loading of the sample, it is expected that the position of a continuous crack tip could be identified by a large local strain concentration.

Images were captured at the load peak, prior to partial unloading, during the fracture toughness test on sample LCD3. The resulting strain distributions in the vicinity on the EDM starter notch, as identified in the top image, are shown in Figure 6 for ten stages of increased loadings. The red contour in images (1)-(5) of Figure 6 indicates a large strain region of greater that $3.6 \%$ is apparent that clearly advances with an increase in load. However no significant strains are seen ahead of this red contour indicating that the region ahead of this contour contains significant damage and can't sustain a load. Images (6)-(10) clearly show the development of a plastic zone suggesting that a continuous crack has developed and that the crack tip is located beyond the discontinuous creep damaged zone and hence can sustain a load. This indicates that the discontinuous creep cracked region extended approximately $9 \mathrm{~mm}$ from the initial EDM notch tip, which is consistent with that measured after break open, given in Table 1.

These results confirm the assumption that the 'tail' region identified in Section 5.1 and Figure 3, where there is little change in fracture resistance for the first apparent $3.5 \mathrm{~mm}$ of crack extension, is due to breaking weak unbroken ligaments along the CCG plane. Beyond this discontinuously creep cracked region, there is no significant creep damage ahead of the crack tip, hence the increase in fracture resistance with crack extension (R-curves behaviour) of the LCD samples is similar to the un-crept PC samples. 
The fracture toughness data analysis has been performed on the R-curves obtained from the tests on LCD1 and LCD2 specimens. The fracture toughness value has been obtained without considering the effects of the discontinuous creep cracked region shown above as this would give a value representative of the resistance expected should a sample be extracted from a highly creep damaged component in-service. Data for specimens LCD1 and LCD2 were therefore analysed according to the standard [18] and all validity checks performed. The slope of the blunting lines and the values of $\Delta a_{\max }, J_{\max }, J_{0.2 / B L}, \Omega, 2 d J / d \Delta a_{(0.2 / B L)}$ and $J_{0.2}$ for these two tests on the LCD samples are summarised in Table 3. It can be seen in this table that the $\Omega$ values obtained from these two tests are smaller than 10, this signifies dependency of the valid data points which fall below $J_{\max }$ on specimen size. Also seen in this table is that $2 d J / d \Delta a_{(0.2 / B L)}$ is smaller than the slope of the blunting line and $J_{0.2 / B L}$ values are smaller than the $J_{\text {max }}$ in both tests on LCD1 and LCD2 specimens. Thus the obtained values of $J_{0.2 / B L}$ for these two tests are considered valid. Furthermore, $J_{0.2}$ values found in these two tests are smaller than $J_{\max }$ and at least one data point falls in between 0.2 and $0.4 \mathrm{~mm}$ vertical crack extension lines for LCD1 and LCD2 specimens. Therefore, $J_{0.2}$ values obtained from these two tests may also be considered valid.

The fracture toughness test results on the LCD samples are compared with those of obtained from the PC specimens in Table 3. The slopes of the blunting lines are assumed the same in the PC and LCD specimens since they are both pre-compressed material. Since the same material properties have been assumed values of $J_{\max }$ are also similar in the LCD and PC specimens. The apparent fracture toughness parameter, $J_{0.2 / B L}$, is seven times less in the LCD specimens than in the PC specimens. However, as indicated by the strain field measurements above, this only corresponds to the energy required to rupture the unbroken ligaments in the material.

\subsection{Fractography on a LCD Specimen}

A detailed micro structural study of the cracking mode in a specimen containing creep damage has been performed by capturing scanning electron microscopic (SEM) pictures on the fracture surface of the LCD2 specimen. These images are shown in Figure 7. As seen in this figure, the cracking mode in the CCG region is intergranular whereas transgranular deformation can be observed in the fracture toughness area. Transgranular voids are clearly visible in the SEM images corresponding to the fracture toughness region. Intergranular deformation is dominant along a line corresponding to the average final creep crack length measured on the fracture surface, though some transgranular void growth can also be observed. This indicates that an interaction between both failure mechanisms may be expected at the interface between the creep cracked and fracture toughness regions, where the unbroken ligaments introduced by CCG are ruptured during the fracture toughness test. At 1 $\mathrm{mm}$ into the fracture toughness region the dominant failure mechanism is transgranular crack growth and plastic void growth is evident but no intergranular creep damage is seen. These images confirm the assumptions made above.

\section{Discussion}

Comparison of the measured values of crack extension during the fracture toughness tests (see Table 2) to those of estimated by the unloading compliance (see Figure 3) shows that similar results are found for JIC-AR1, JIC-AR2, JIC-PC1 and JIC-PC2 specimens. This implies that Eqn (1) provides good estimates of the crack length in fracture toughness tests on AR and PC Type $316 \mathrm{H}$ stainless steel, where no creep damage is present. However, crack growth estimates from the compliance technique during the fracture toughness test (see Figure 3 ) on the LCD specimens have been found greater than that measured from the ductile 
fracture surface (see Figure 7 and Table 2) and less than the summation of the CCG and ductile fracture surface. This discrepancy is due the fact that the location of a continuous crack tip in the LCD samples is uncertain. Hence, during the fracture toughness test, crack extension initiates somewhere in the CCG region where the unbroken ligaments exist, and the final crack length is beyond the creep damage region.

The compliance technique for crack length measurement is based on a single straight fronted crack. Hence, crack length measurements in the creep damaged region, which contains a large number of small discontinuous cracks, obtained from the compliance technique may therefore be inaccurate. In order to examine the accuracy of the crack length estimates from the unloading compliance data in the presence of discontinuous micro cracks, the crack length measurements from the DIC test data on the plain sided LCD specimen (see Figure 6) are plotted against the crack length estimates from the unloading compliance in Figure 8. Note that the DIC crack length measurements on the surface of the plain sided LCD specimen was only taken up to the end of CCG region where discontinuous micro cracks exist, beyond which the crack length estimates in the homogenous material with minor creep damage may be considered relatively accurate. Figure 8 shows that although some discrepancy can be observed in the DIC crack length measurements on the surface and those of estimated from the unloading compliance in the LCD specimen, the percentage error in these estimates within the CCG region was found on average around 3\%. This percentage error may be considered small and thus the crack growth estimates from the unloading compliance data in the CCG region of the LCD1 and LCD2 specimens shown in Figure 3 may be considered reasonably accurate.

The appearance of the tail region in the R-curve of the LCD specimens in Figure 3 shows that the creep damage introduced into the material weakens it and thus little energy is needed to rupture the unbroken ligaments. It must be noted that in order to observe the tail region in fracture toughness behaviour of the LCD specimen, small displacement intervals similar to that of shown in Figure 3 need to be employed. An instant increase in the applied load without any unloading at the early stage of the test may cause the unbroken ligaments to suddenly rupture, in which case the tail region may not be seen.

The LCD sample results may be representative of components containing significantly large macro-cracks. However in order to better represent the condition of uncracked components that contain a significant amount of creep strain and damage, an alternative sample design is proposed where fracture samples are extracted from a large uniaxial geometry that has been crept uniformly to a critical life/strain fraction. This alternative approached, denoted the global creep damage method, is expected to overcome some of the difficulties encountered in the LCD fracture toughness tests.

\section{Conclusions}

Creep damage local to the crack tip was introduced into the material by interrupting CCG tests on $\mathrm{C}(\mathrm{T})$ specimens at $550{ }^{\circ} \mathrm{C}$. To limit the extent of local plasticity ahead of the crack tip in CCG tests, the material was uniformly pre-compressed to $8 \%$ plastic strain at room temperature prior to specimen manufacturing, thus hardening the material. Room temperature fracture toughness tests were performed on AR, PC and LCD specimens. An additional test on a plain sided LCD specimen was also performed in conjunction with DIC measurements to visualise the crack growth behaviour, particularly at early stages of the test. Larger LLD in the fracture toughness test data on the AR material was observed compared to the PC and LCD specimens. A drop in fracture toughness values was seen in the PC material compared to the AR. A tail region was observed in the resistance curves of the LCD specimens and significantly smaller values of crack growth resistance were found in these specimens 
compared to the PC material with no creep damage. The DIC results confirmed that the appearance of a tail region and the low fracture toughness in LCD specimens are mainly due to the existence of discontinuous intergranular micro cracks in the CCG region which linked up during the early stages of the fracture toughness tests. It can be concluded from the fracture toughness test results that the inelastic damage (combined plastic pre-straining and creep damage) reduces the fracture toughness of the material, however the drop in fracture toughness due to creep damage is a lot more severe than uniform plastic pre-straining.

\section{Acknowledgements}

The authors would like to thank Mr Roger Smith and Mr David McLennon from EDF Energy for their technical assistance with fracture toughness testing. This work has been supported by EDF Energy and EPSRC under grant EP/I004351/1.

\section{References}

1. Amouzouvi, K. F., A comparative fracture study of a slightly prestrained low alloy steel and a slightly prestrained austenitic stainless steel, Materials Science and Engineering, 1986, 78(1), pp. 65-70.

2. Liaw, P. K. and Landes, J. D., Influence of prestrain history on fracture toughness properties of steels, Metallurgical and materials transactions. A, Physical metallurgy and materials science, 1986, 17(3), pp. 473-489.

3. Sivaprasad, S., Tarafder, S., Ranganath, V. R. and Ray, K. K., Effect of prestrain on fracture toughness of HSLA steels, Materials Science and Engineering: A, 2000, 284(1-2), pp. 195-201. http://dx.doi.org/10.1016/S0921-5093(00)00739-5

4. Fukuda, N., Hagiwara, N. and Masuda, T., Effect of Prestrain on Tensile and Fracture Toughness Properties of Line Pipes, Journal of Offshore Mechanics and Arctic Engineering, 2005, 127(3), pp. 263-268. 10.1115/1.1894405

5. Hagiwara, N., Oguchi, N. and Masuda, T., Effects of Prestrain on Fracture Toughness and FatigueCrack Growth of Line Pipe Steels, Journal of Pressure Vessel Technology, 2001, 123(3), pp. 355-361. 10.1115/1.1379531

6. Minami, F. and Arimochi, K., Evaluation of Prestraining and Dynamic Loading Effects on the Fracture Toughness of Structural Steels by the Local Approach, Journal of Pressure Vessel Technology, 2001, 123(3), pp. 362-372. 10.1115/1.1379532

7. Mills, W. J., Fracture toughness of type 304 and 316 stainless steels and their welds, International Materials Reviews, 1997, 42(2), pp. 45-82. doi:10.1179/imr.1997.42.2.45

8. Ainsworth, R. A., An assessment of the effects of prestrain on upper shelf fracture toughness, The Journal of Strain Analysis for Engineering Design, 1986, 21(4), pp. 219-224. $10.1243 / 03093247 \mathrm{v} 214219$

9. Gan, D., Tensile and fracture properties of type 316 stainless steel after creep, Metallurgical and Materials Transactions A, 1982, 13(12), pp. 2155-2163. 10.1007/bf02648385

10. Albertini, C. and Montagnani, M., Strain-rate dependence of residual strength and ductility of AISI 316 stainless steel after creep, fatigue and irradiation, Res mechanica, 1990, 30(4), pp. 361-75.

11. Mehmanparast, A., Davies, C. M., Dean, D. W. and Nikbin, K. M., The Influence of Inelastic Damage on Tensile Deformation and Creep Crack Growth Behaviour of Type 316H Stainless Steel, in ASMEPVP 14-18 July 2013, Proceedings of the International Conference on Pressure Vessels and Piping: Paris-France

12. Moskovic, R., Lingham, I. J., Crocker, A. G., Smith, G. E. and Flewitt, P. E. J., An experimental and theoretical consideration of the effect of prior creep damage on the heat affected zone fracture toughness of CrMoV steel, Engineering Fracture Mechanics, 2004, 71(4-6), pp. 587-599. http://dx.doi.org/10.1016/S0013-7944(03)00028-6

13. Smith, G. E., Crocker, A. G., Flewitt, P. E. J. and Moskovic, R., Effect of prior creep cavitation on brittle fracture in heat affected zone of ferritic steel weldments, Materials Science and Technology, 2002, 18(11), pp. 1329-1334. doi:10.1179/026708302225006070

14. Mehmanparast, A., Davies, C. M., Dean, D. W. and Nikbin, K. M., The Influence of Pre-Compression on the Creep Deformation and Failure Behaviour of Type $316 \mathrm{H}$ Stainless Steel, Engineering Fracture Mechanics, 2013, 110, pp. 52-67. 10.1016/j.engfracmech.2013.08.006

15. Anderson, T. L., Fracture Mechanics: Fundamentals and Application, 1991, Boston: CRC Press. 
16. Rice, J. R. and Tracey, D. M., On the Ductile Enlargement of Voids in Triaxial Stress Fields, Journal of Mechanics and Physical Solids, 1969, 17, pp. 201-217.

17. ASTM, E 1820-09: Standard Test Method for Measurement of Fracture Toughness, Annual Book of ASTM Standards, 2009, 03.01, pp. 1024-1057.

18. ESIS, P2-92: Procedure for Determining the Fracture Behaviour of Materials, 1992, European Structural Integrity Society Publication.

19. Davies, C. M., Kourmpetis, M., O'Dowd, N. P. and Nikbin, K. M., Experimental Evaluation of the J or $C^{*}$ Parameter for a Range of Cracked Geometries, Journal of ASTM International, 2006, 3(4), DOI: 10.1520/JAI13220.

20. Dean, D. W. and Gladwin, D. N., Creep Crack Growth Behaviour of Type 316H Steels and Proposed Modifications to Standard Testing and Analysis Methods, International Journal of Pressure Vessels and Piping, 2007, 84(6), pp. 378-395.

21. Tarnowski, K., Davies, C. M., Nikbin, K. M. and Dean, D. W., The influence of strain on crack length measurements using the potential drop technique, in 16th International Conference on Experimental Mechanics 7-11 July. 2014: Cambridge, UK

22. Mehmanparast, A., Davies, C. M., Wimpory, R. C. and Nikbin, K. M., Quantification of Residual Stresses Induced by Prior Loading at High Temperatures, in ASME-PVP 17-21 July 2011, proceedings of the International Conference on Pressure Vessels and Piping: Baltimore-US

23. Mehmanparast, A., Davies, C. M. and Nikbin, K. M., Quantification and Prediction of Residual Stresses in Creep Crack Growth Specimens, Materials Science Forum, 2014, 777, pp. 25-30. 10.4028/www.scientific.net/MSF.777.25 


\section{Tables}

Table 1: Creep crack length measurements in LCD specimens subsequent to interruption

\begin{tabular}{|c|c|}
\hline Test ID & $\begin{array}{c}a_{c} \\
(\mathrm{~mm})\end{array}$ \\
\hline LCD1 & 27.1 \\
\hline LCD2 & 27.7 \\
\hline LCD3 & 26.3 \\
\hline
\end{tabular}

Table 2: Fracture toughness specimen dimensions

\begin{tabular}{|c|c|c|c|c|c|c|}
\hline Test ID & $\begin{array}{c}W \\
(\mathrm{~mm})\end{array}$ & $\begin{array}{c}B \\
(\mathrm{~mm})\end{array}$ & $\begin{array}{c}B_{n} \\
(\mathrm{~mm})\end{array}$ & $\begin{array}{c}a_{i} \\
(\mathrm{~mm})\end{array}$ & $\begin{array}{c}a_{f} \\
(\mathrm{~mm})\end{array}$ & $\begin{array}{c}\Delta a \\
(\mathrm{~mm})\end{array}$ \\
\hline JIC-AR1 & 50.0 & 25.0 & 20.1 & 25.3 & 28.1 & 2.8 \\
\hline JIC-AR2 & 50.1 & 25.0 & 20.0 & 26.0 & 29.3 & 3.3 \\
\hline JIC-PC1 & 50.1 & 25.0 & 20.0 & 26.6 & 29.5 & 2.9 \\
\hline JIC-PC2 & 50.0 & 25.0 & 20.0 & 24.7 & 27.3 & 2.6 \\
\hline LCD1 & 50.0 & 25.0 & 17.5 & 27.1 & 30.4 & 3.3 \\
\hline LCD2 & 50.0 & 25.0 & 17.5 & 27.7 & 31.9 & 4.2 \\
\hline
\end{tabular}

Table 3: A summary of the fracture toughness test results

\begin{tabular}{|c|c|c|c|c|c|c|c|}
\hline Test ID & $\begin{array}{l}\Delta a_{\max } \\
(\mathrm{mm})\end{array}$ & $\begin{array}{c}J_{\max } \\
(\mathrm{MPam})\end{array}$ & $\begin{array}{c}\text { Blunting line } \\
\text { slope }\end{array}$ & $\Omega$ & $\begin{array}{c}J_{0.2 / B L} \\
(\mathrm{MPam})\end{array}$ & $2 d J / d \Delta a_{(0.2 / B L)}$ & $\begin{array}{c}J_{0.2} \\
(\mathrm{MPam})\end{array}$ \\
\hline JIC-AR1 & 2.48 & 0.57 & \multirow{2}{*}{2.334} & 36.53 & 0.47 & 1.73 & 0.29 \\
\hline JIC-AR2 & 2.41 & 0.55 & & 24.38 & 0.25 & 1.24 & 0.18 \\
\hline JIC-PC1 & 2.34 & 0.50 & \multirow{4}{*}{2.333} & 13.63 & 0.21 & 0.76 & 0.17 \\
\hline JIC-PC2 & 2.53 & 0.54 & & 11.74 & 0.21 & 0.83 & 0.17 \\
\hline LCD1 & 2.29 & 0.49 & & 6.34 & 0.03 & 0.08 & 0.03 \\
\hline $\mathrm{LCD} 2$ & 2.24 & 0.48 & & 8.53 & 0.03 & 0.07 & 0.03 \\
\hline
\end{tabular}


Figures

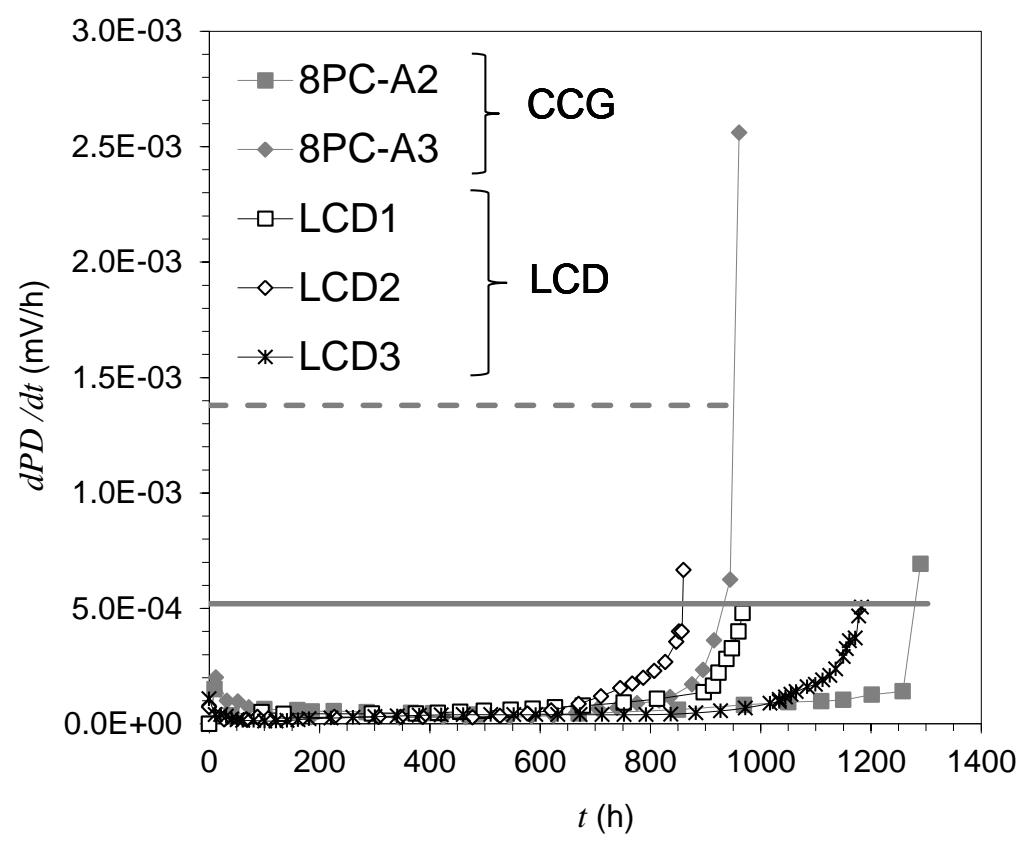

Figure 1: Comparison of the PD rates in LCD and CCG calibration tests

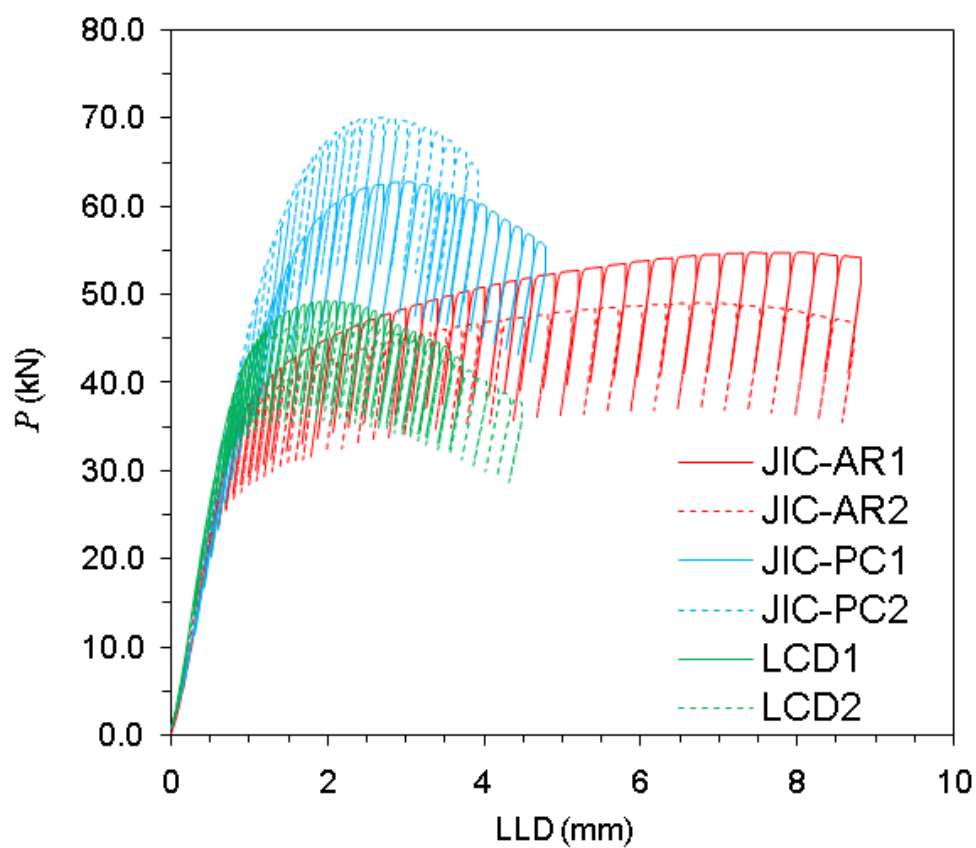

Figure 2: Load vs. LLD data for the fracture toughness tests performed on the AR, PC and LCD specimens 


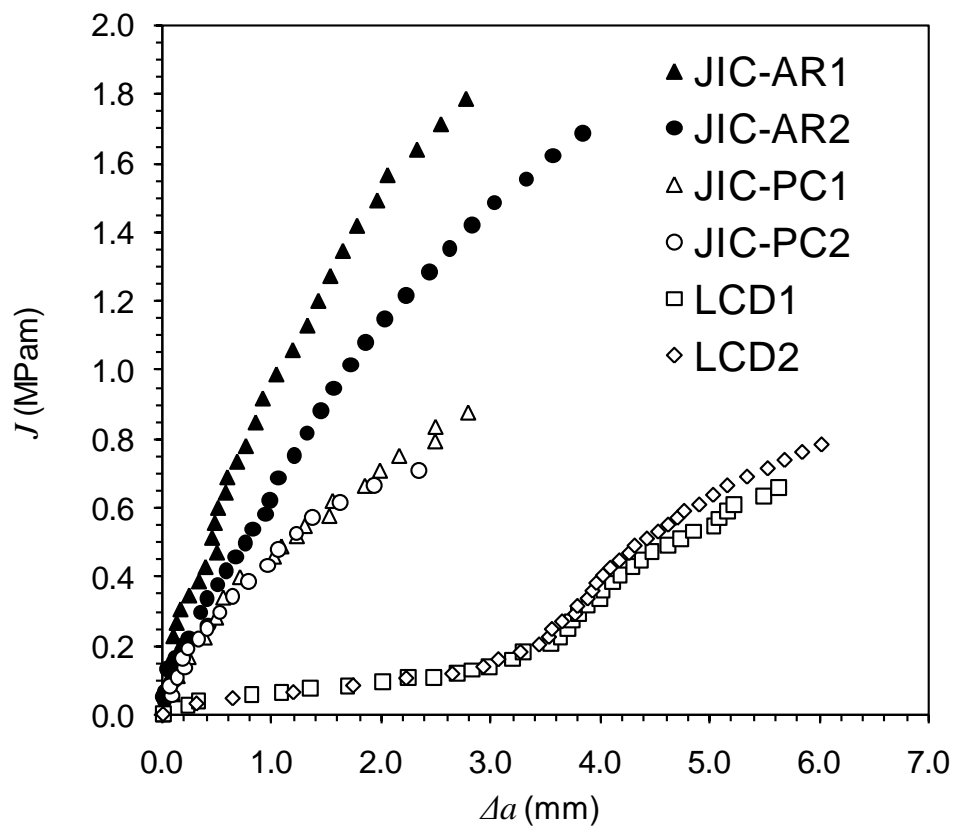

Figure 3: The fracture resistance curves for the AR, PC and LCD specimens
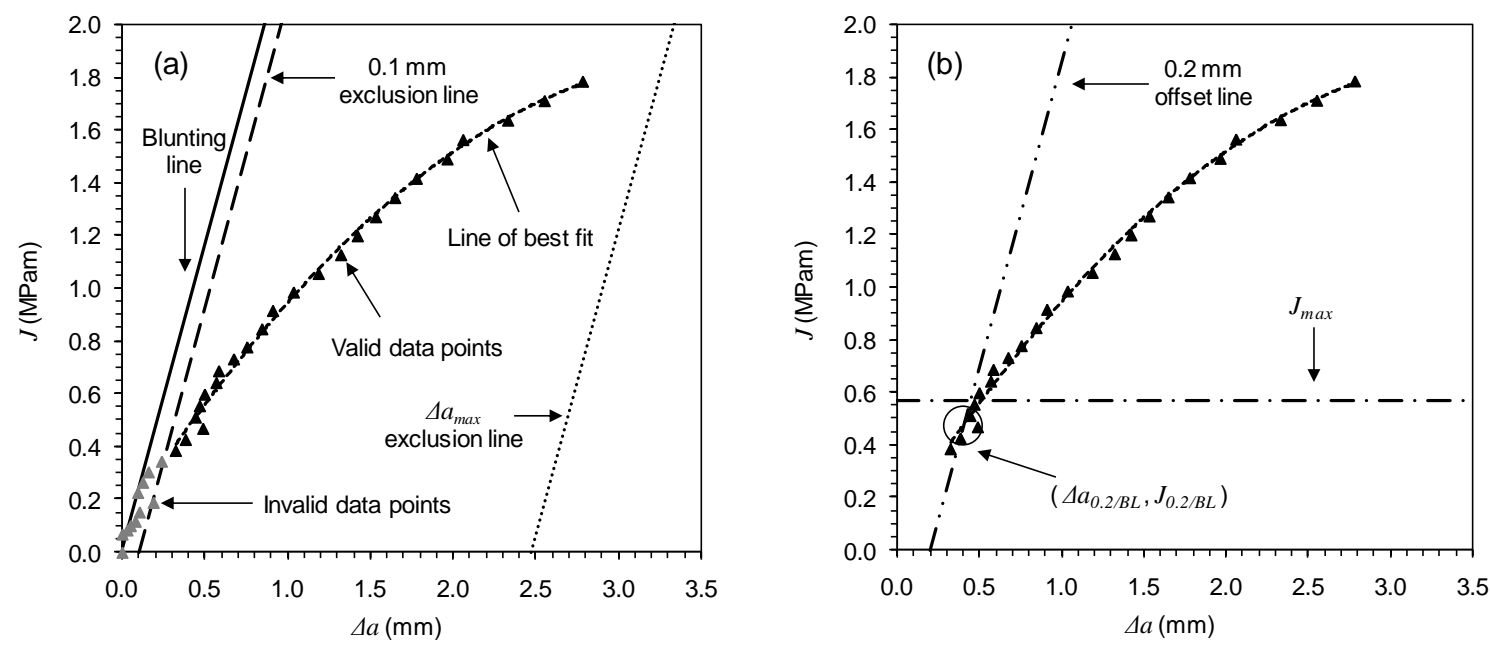

Figure 4: Fracture toughness data analysis on JIC-AR1 specimen (a) illustration of the exclusion lines (b) calculation of $J_{0.2 / B L}$

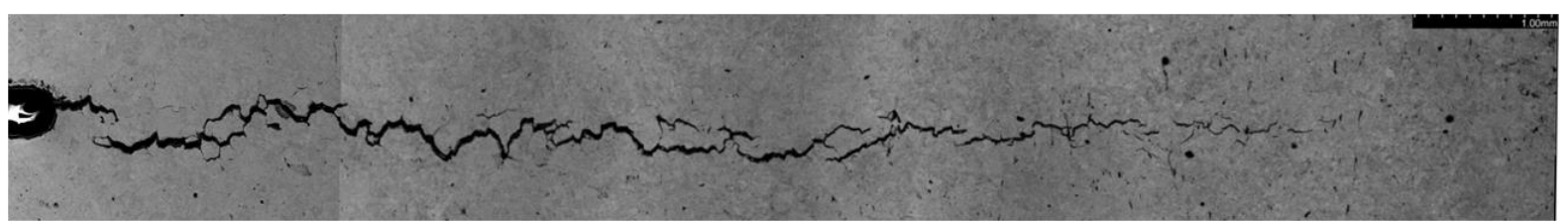

Figure 5: Creep crack growth behaviour in a PC specimen [14] 

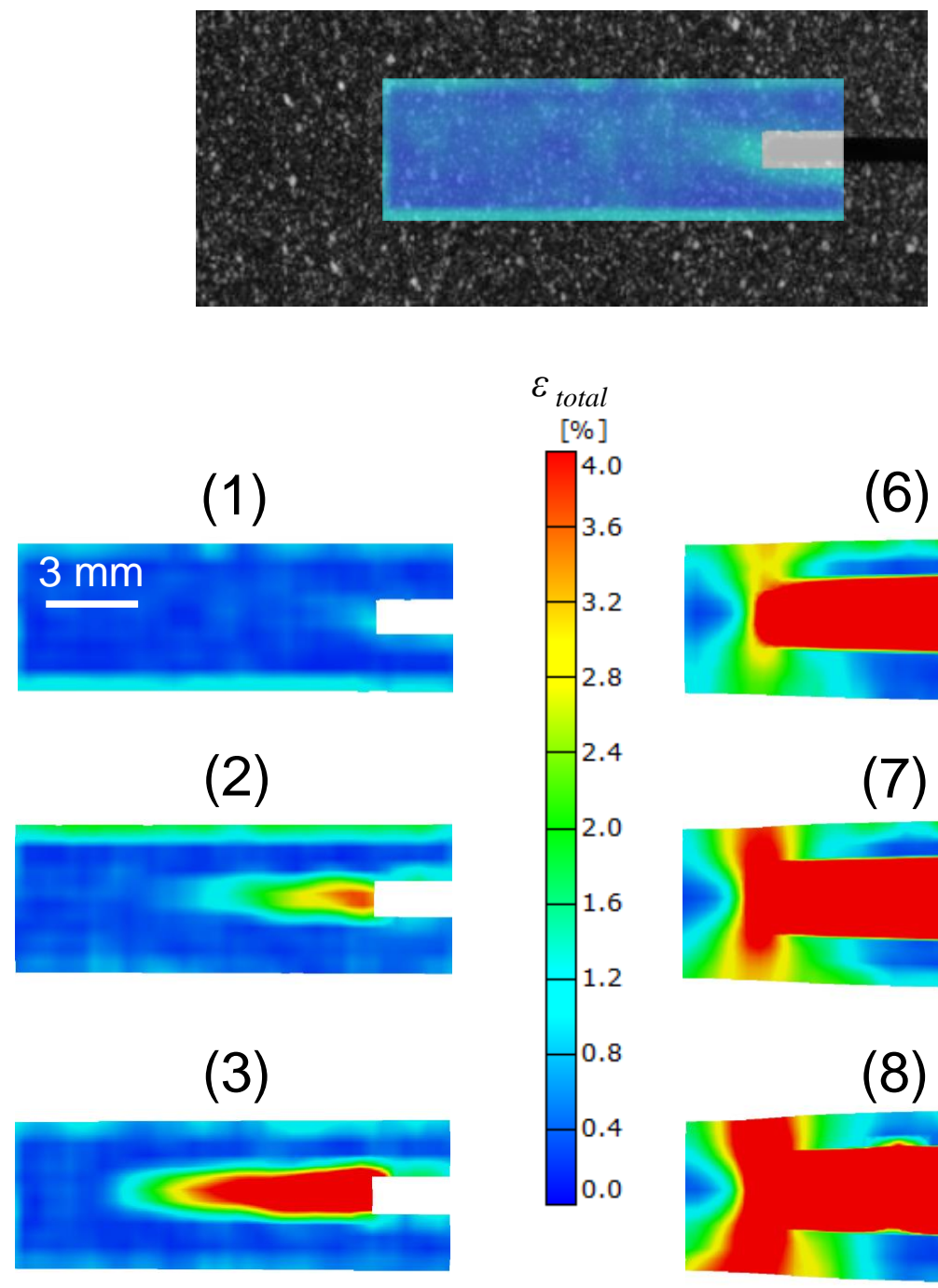

(6)

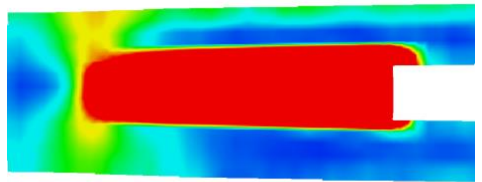

(7)

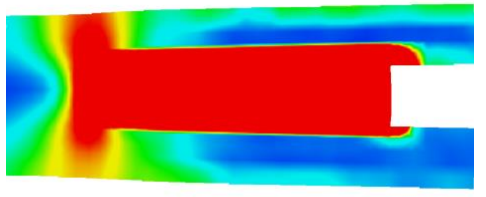

(8)

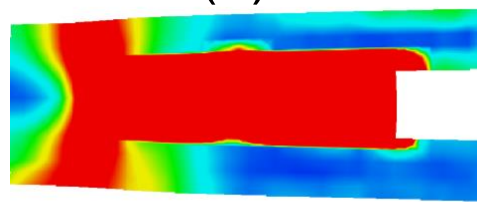

(4)

(9)
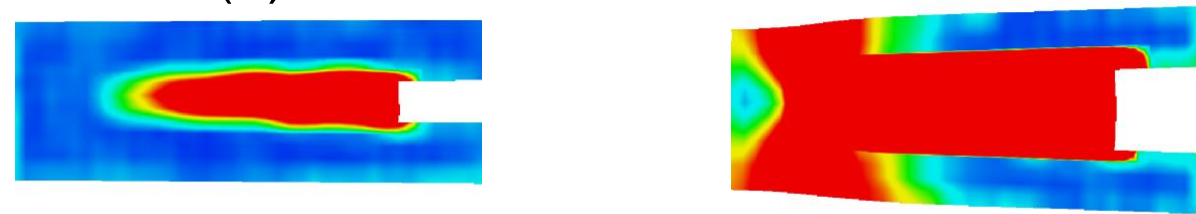

(5)

(10)
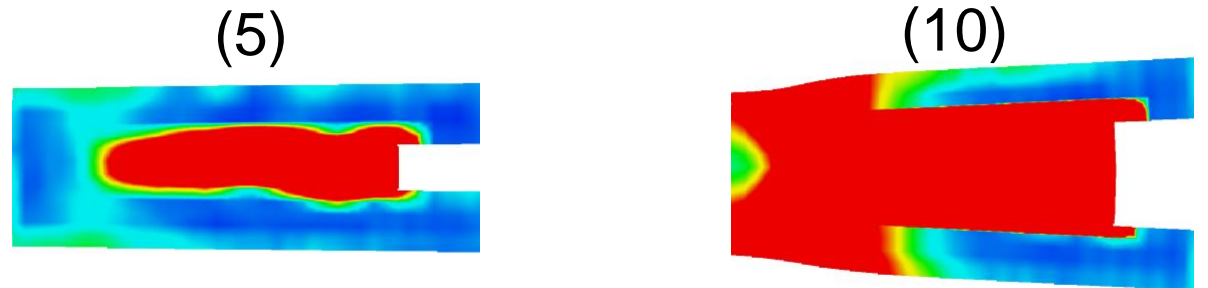

Figure 6: The strain distribution in the fracture toughness test on the plain sided LCD3 specimen obtained from the DIC measurements 


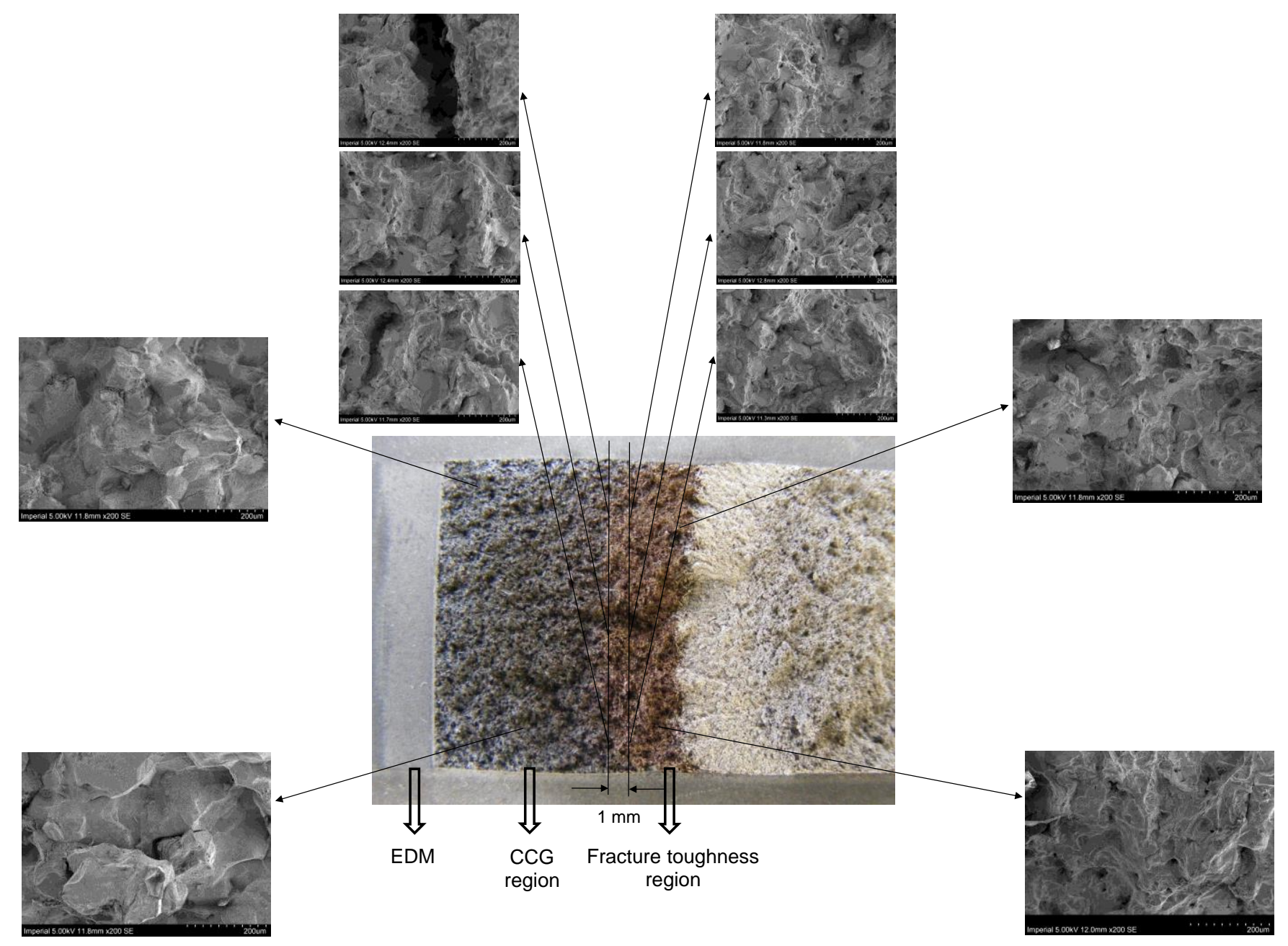

Figure 7: SEM study of the failure mechanisms on the fracture surface of the LCD2 specimen 


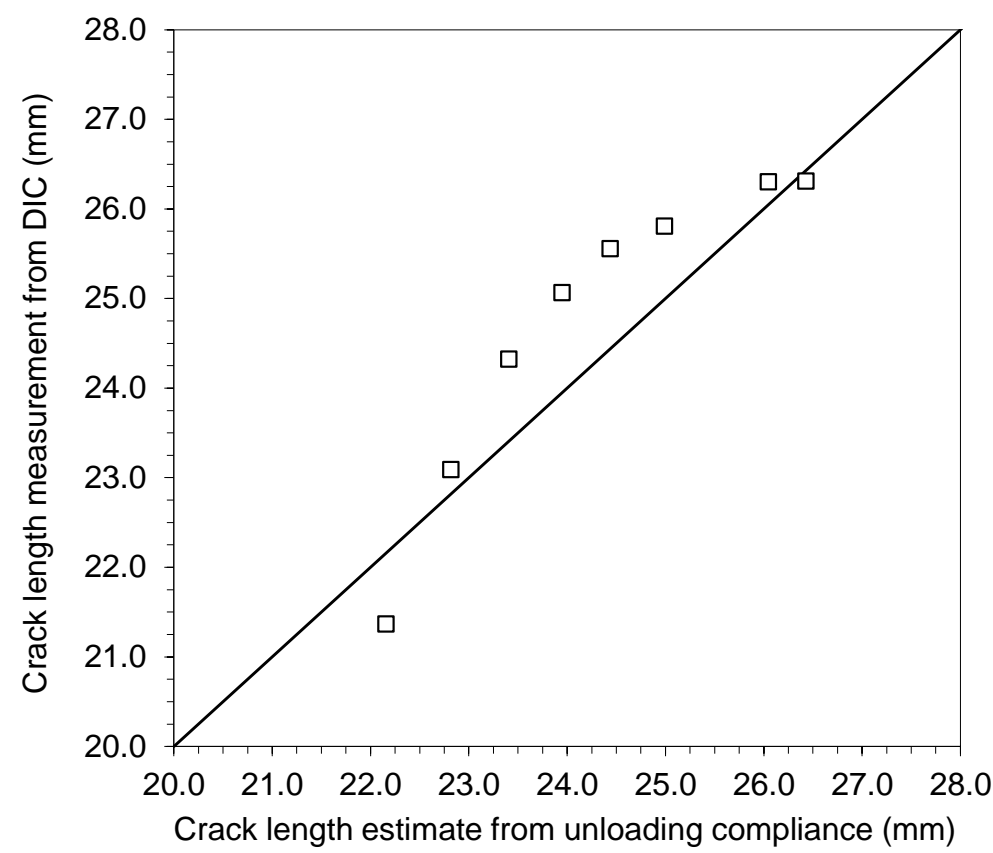

Figure 8: Comparison of the crack length measurements from a DIC test on a plain sided LCD specimen with those of estimated from the unloading compliance 